cross-section for some value of the angle of collision, and the results in the remaining angular domain are examined. This permits us to see the angular dependence of the cross-section for each kind of field. (ii) It also seems useful to try to relate the results at low energies with those at higher energies, in looking for the role of the relativistic corrections in the total cross-section ${ }^{3}$. Using, for example, a mixture of symmetrical pseudoscalar and vector fields, we get for the total cross-section, in the system of the centre of gravity, and neglecting terms of the fourth order in the momentum $p$ of nucleons :

$$
\begin{aligned}
S=S_{N R}(1 & \left.-\frac{p^{2}}{M^{2}}\right)+\frac{p^{2}+M^{2}}{8 \pi}\left\{\frac{10 A}{\varkappa^{2}\left(x^{2}+4 p^{2}\right)}\right. \\
& -\frac{5 A^{\prime}}{4 p^{2}}\left[\frac{1}{p^{2}} \log \frac{\varkappa^{2}+4 p^{2}}{\varkappa^{2}}-\frac{4}{\varkappa^{2}} \frac{\varkappa^{2}+2 p^{2}}{\chi^{2}+4 p^{2}}\right] \\
& \left.+\frac{B}{p^{2}\left(\varkappa^{2}+2 p^{2}\right)} \log \frac{\varkappa^{2}+4 p^{2}}{\varkappa^{2}}\right\} \frac{p^{2}}{M^{2}}
\end{aligned}
$$

with

$$
\begin{aligned}
& A=g_{1}{ }^{3}\left(g_{1}\right.\left.-4 g_{2} \frac{M}{\varkappa}\right) \\
&+g_{2}{ }^{2}\left(4 g_{2}{ }^{2} \frac{M^{2}}{\varkappa^{2}}-f_{3}{ }^{2}-2 g_{1}{ }^{2}+8 g_{1} g_{2} \frac{M}{\varkappa}\right) \\
& A^{\prime}=g_{1}{ }^{4}+ g_{2}{ }^{4}+g_{1}{ }^{3}\left(g_{1}-4 g_{2} \frac{M}{\varkappa}\right) \\
&-g_{2}{ }^{2}\left(4 g_{2}{ }^{2} \frac{M^{2}}{\chi^{2}}-f_{3}{ }^{2}-2 g_{1}{ }^{2}+8 g_{1} g_{2} \frac{M}{\varkappa}\right) \\
& B=g_{1}{ }^{2}\left(4 g_{2}{ }^{2} \frac{M^{2}}{\chi^{2}}-f_{3}{ }^{2}-g_{1}{ }^{2}+4 g_{1} g_{2} \frac{M}{\varkappa}\right) \\
& \quad-g_{2}{ }^{2}\left(4 g_{2}{ }^{2} \frac{M^{2}}{\varkappa^{2}}-f_{3}{ }^{2}-5 g_{1}{ }^{2}+20 g_{1} g_{2} \frac{M}{\varkappa}\right) \\
& \quad+8 g_{1} g_{2} \frac{M}{\varkappa}\left(g_{1}{ }^{2}+3 g_{2}{ }^{2}\right),
\end{aligned}
$$

where $g_{1}, g_{2}$ are the coupling constants between the vector meson field and the nucleons.

The corresponding non-relativistic cross-section is :

$$
\begin{array}{r}
S_{N R}=\frac{p^{2}+M^{2}}{8 \pi}\left\{\left(g_{1}{ }^{4}+3 g_{2}{ }^{4}\right) \frac{10}{\varkappa^{2}\left(\varkappa^{2}+4 p^{2}\right)}+\right. \\
\left.\frac{g_{1}^{4}-3 g_{2}^{4}+6 g_{1}^{2} g_{2}{ }^{2}}{p^{2}\left(\varkappa^{2}+2 p^{2}\right)} \log \frac{\varkappa^{2}+4 p^{2}}{\varkappa^{2}}\right\}
\end{array}
$$

and it does not involve any pseudoscalar coupling constants.

Using, for example, the constants of interaction of the Møller-Rosenfeld theory, $f_{1}=g_{1}=0.62 ; f_{2}=$ $g_{2}=1.08$, corresponding to a meson mass $286^{2} m_{e}$, one gets, for incident neutrons of $90 \mathrm{MV}$. (laboratory system), $\quad S_{N R}=13.5 \times 10^{-26} \mathrm{~cm}^{2}$. The experimental value? is approximately $9 \times 10^{-26} \mathrm{~cm} .{ }^{2}$. The non-relativistic cross-section, as in the usual theories, is too large. If we take into account the relativistic terms, we obtain $S=16.2 \times 10^{-28} \mathrm{~cm} .^{2}$, showing a further increase in the cross-section. This result is not changed by a more exact evaluation of the crosssection in terms of the velocities of the nucleons. In order to draw more definite conclusions, however, it seems necessary to revise the usual treatment of the ground state of the deuteron by taking account of the velocity-dependent and contact interactions.

I wish to thank Prof. L. Rosenfeld for many valuable discussions, and am also indebted to the
Centre National de la Recherche Scientifique for a grant which entitled me to stay at the University of Manchester.

Physics Department,

C. MARTY University,

Manchester 13.

1 Van Hove, L., Phys. Rev., 75, 1519 (1949).

${ }^{3}$ Araki, G., Phys. Rev., 75, 1101, 1262 (1949).

s Snyder, H., and Marshak, R. E., Phys. Rev., 72, 1253 (1945).

AAlzofon, F. E., Phys. Rev., 75, 1773 (1949).

S Kemmer, N., Helv. Phys. Acta, 10, 47 (1937).

- Rosenfeld, L., Kgl. Danske Vid., 23, No. 13 (1945).

' Cook, L. J., McMillan, E. M., Peterson, J. M. and Sewell, D. C., Segrè, E., Wiegand, C., and York, H., Phys. Rev., 75, 351 (1949).

\section{Meyer Analysis of Metals}

Messrs. Finniston, Jones and Madsen discuss in a recent communication ${ }^{1}$ the variation of ultimate hardness number $P u$ with the Meyer index $n$, in the ball indentation test. There are several good reasons for associating a decrease in the $n$ value with a decrease in the work-hardening capacity of a metal, especially since $n$ and $P u$ have hitherto shown a negative correlation coefficient. Finniston et al., however, suggest that this generalization should be reconsidered, and they give results showing a positive correlation coefficient between $P u$ and $n$ for metals of non-cubic structure and of increasing anisotropy as judged by results for thermal expansion. Their proposal is interesting because certain anomalies exist regarding the $n$ value. Thus, during the progressive cold rolling of copper it may drop from $2 \cdot 34$ to 2.0 at an early stage and then remain more or less constant, while the $P u$ value will continue to increase and thus indicate further work-hardening ${ }^{2}$. This peculiarity has not boen explained, and it should be remarked that the progressively rolled copper increasingly develops anisotropic properties (preferred orientation) as shown by X-ray diffraction and etching tests. This effect does not appear to be inconsistent with their proposal.

Furthermore, there is evidence that when a stainless steel $18 / 8$ alloy is progressively cold-worked, the $P u$ value rises steadily while $n$ first falls and then rises. During the tempering of high-carbon steel, $P u$ will fall progressively but $n$ will first fall and then rise slightly. Finally, in the course of investigations with the new hardness microtesters, results are being obtained where the Meyer index to the pyramid indentation increases with cold working as compared with a fall in the normal ball test.

\section{University College, \\ Swansea.}

Jan 16.

${ }^{1}$ Nature, 164, 1128 (1949).

2O'Neill and Cuthbertson, J. Inst. Metals, No. 2 (1931).

\section{The Geminid Meteor Shower}

IN 1947, Whipple ${ }^{1}$ published new elements of the Geminid meteor shower, obtained photographically. An extremely short period, 1.65 years, moderate inclination and considerable eccentricity (see below) together make the orbit of this shower an extraordinary one both in comparison with comets and with minor planets. But, according to Hoffmeister ${ }^{2}$, the existence of similar meteor showers seems to be indicated. Such a short-period meteor shower as 